\title{
Periodic solutions to the self-dual Ginzburg-Landau equations
}

\author{
Y. ALMOG† \\ Department of Applied Mathematics and Computer Science, The Weizmann Institute of Science, \\ Rehovot 76100, Israel
}

(Received 30 April 1998; revised 12 February 1999)

\begin{abstract}
The structure of periodic solutions to the Ginzburg-Landau equations in $\mathbb{R}^{2}$ is studied in the critical case, when the equations may be reduced to the first-order Bogomolnyi equations. We prove the existence of periodic solutions when the area of the fundamental cell is greater than $4 \pi M, M$ being the overall order of the vortices within the fundamental cell (the topological invariant). For smaller fundamental cell areas, it is shown that no periodic solution exists. It is then proved that as the boundaries of the fundamental cell go to infinity, the periodic solutions tend to Taubes' arbitrary $N$-vortex solution.
\end{abstract}

\section{Introduction}

Planar periodic solutions to the Ginzburg-Landau equations were first predicted in 1957 by Abrikosov [1], who studied their linear bifurcation from the normal state. A linear bifurcation analysis of different periodic structures was performed by Kleiner et al [2], who found their solution to be energetically preferable to that of Abrikosov [1]. Chapman [3] studied the linear bifurcation of several other periodic structures together with the linear stability of Abrikosov's [1] solution, which was found unstable, and of the solution in Kleiner et al [2], which was found stable to several different modes of perturbations. The periodic structure in [2] has also been observed experimentally [4].

Rigorous analysis of periodic solutions to the Ginzburg-Landau equations in the highly non-linear regime is, presently, quite limited. Odeh [5] proved the existence of such solutions near their bifurcation from the normal state, where the equations can be linearized. In the highly non-linear regime he proved, using direct methods [5], the existence of weak (variational) periodic solutions for fundamental cell areas which exceed a certain critical value. Odeh's method cannot, however, provide information either on the possible structure of solutions inside the fundamental cell, or on their energy. Several conjectures [6] are associated, however, with the behaviour of solutions in the highly nonlinear regime. Therefore, further analysis of solutions in that regime may be physically valuable.

The present contribution focuses on the critical case, when the Ginzburg-Landau parameter $\kappa=1 / \sqrt{2}$. It is well known [7] that in this case the Ginzburg-Landau equations may be reduced to a system of first order equations. This phenomenon is often

$\dagger$ Present address: Faculty of Mathematics, Technion-IIT, Haifa 32000, Israel 
studied within the framework of Yang-Mills Theory in Mathematical Physics. Arbitrary $N$-vortex solutions tending as $|x| \rightarrow \infty$, either to the perfectly superconducting state [8], or to the normal state [9], have already been studied. Such solutions have also been studied on compact Riemann surfaces [10] and on asymptotically Euclidean Riemann manifolds [11].

We study here the structure of periodic solutions in the critical case. We first rederive the results in [10] using a different method: we prove the existence, for sufficiently large fundamental cell of periodic solutions and demonstrate their non-existence for smaller fundamental cells. Though we consider only periodic solutions in $\mathbb{R}^{2}$ (or equivalently on a torus), our results can be easily generalized to the same class of Riemann surfaces discussed in Noguchi [10]. Our method is proved later to be constructive when we investigate the behaviour of solutions in the large cell limit. With the aid of the iterative scheme we have developed (the proof in Noguchi [10] employs direct methods of the calculus of variations), we derive some estimates which enable us to show that in the large cell limit the periodic solutions tend to the arbitrary $N$-vortex solution in $\mathbb{R}^{2}$. Thus, this limit relates the solutions on compact Riemann manifolds with the arbitrary $\mathrm{N}$-vortex solutions in the plane, a relation which is not obvious in Yang-Mills theory.

The steady state Ginzburg-Landau equations, can be written in the following dimensionless form [3]:

$$
\begin{aligned}
& \left(\frac{i}{\kappa} \nabla+\boldsymbol{A}\right)^{2} \psi=\psi\left(1-|\psi|^{2}\right), \\
& -\nabla \times(\nabla \times \boldsymbol{A})=\frac{i}{2 \kappa}\left(\psi^{*} \nabla \psi-\psi \nabla \psi^{*}\right)+|\psi|^{2} \boldsymbol{A},
\end{aligned}
$$

in which $\psi$ is the (complex) superconducting order parameter, such that $|\psi|$ varies from $|\psi|=0$ (when the material is at a normal state) to $|\psi|=1$ (for the purely superconducting state). The magnetic vector potential is denoted by $\boldsymbol{A}$ (so that the magnetic field is given by $\boldsymbol{H}=\nabla \times \boldsymbol{A}$ ), and $\kappa$ is the Ginzburg-Landau parameter which is a material property. Superconductors for which $\kappa<1 / \sqrt{2}$ are termed type I superconductors, and those for which $\kappa>1 / \sqrt{2}$ have been termed type II.

For $\kappa=1 / \sqrt{2}$ the Ginzburg-Landau equations exhibit what is known as self-duality. Some of the solutions of $(1.1 a)$ and $(1.1 b)$ may be obtained, in that case, by solving the following system of partial differential equations $[7,8]$ :

$$
\begin{gathered}
\sqrt{2} \frac{\partial \psi_{1}}{\partial x}+A_{x} \psi_{2}-\sqrt{2} \frac{\partial \psi_{2}}{\partial y}+A_{y} \psi_{1}=0 \\
\sqrt{2} \frac{\partial \psi_{1}}{\partial y}+A_{y} \psi_{2}+\sqrt{2} \frac{\partial \psi_{2}}{\partial x}-A_{x} \psi_{1}=0 \\
H+\frac{1}{\sqrt{2}}\left(\psi_{1}^{2}+\psi_{2}^{2}-1\right)=0 .
\end{gathered}
$$

Here, $\psi_{1}$ and $\psi_{2}$ respectively denote the real and imaginary parts of $\psi, A_{x}$ and $A_{y}$ are, respectively, the components of $\boldsymbol{A}$ in the $x$ and $y$ directions, and $H$ denotes the magnetic field magnitude. It is possible to reduce (1.2) into a scalar, second order, elliptic, non-linear 
partial differential equation. Towards this end we set (as in Taubes [8])

$$
\begin{gathered}
\hat{A}=A_{x}+i A_{y} ; \quad \frac{\partial}{\partial z}=\frac{1}{2}\left(\frac{\partial}{\partial x}-i \frac{\partial}{\partial y}\right) \\
\frac{\partial}{\partial z^{*}}=\frac{1}{2}\left(\frac{\partial}{\partial x}+i \frac{\partial}{\partial y}\right) .
\end{gathered}
$$

Equation (1.2a) and (1.2b) may then be written in the form of a single, complex-valued equation

$$
2 \sqrt{2} \frac{\partial \psi}{\partial z^{*}}-i \hat{A} \psi=0
$$

which may be readily solved for $\hat{A}$ to obtain

$$
\hat{A}=-2 \sqrt{2} i \frac{\partial \log \psi}{\partial z^{*}} .
$$

The magnetic field can thus be presented in the form

$$
H=-\sqrt{2} \nabla^{2} f
$$

wherein $f=\log |\psi|$. Combining (1.6) and (1.2c), we obtain

$$
\nabla^{2} f+\frac{1}{2}\left(1-e^{2 f}\right)=0
$$

In the next section we prove the existence of periodic solutions to (1.7) when the fundamental cell area exceeds a certain critical value, and derive their non-existence for smaller fundamental cells. In $\S 3$ the behaviour of periodic solutions in the large cell limit is investigated. In the last section we summarize the main results and discuss several additional points.

\section{Existence of periodic solutions}

In this section we demonstrate the existence of periodic solutions to (1.7). Explicitly, we prove the following theorem:

Theorem 1 Let $R\left(l_{x}, l_{y}\right)$ denote the open rectangle $R=\left\{(x, y) \mid-l_{x} / 2<x<l_{x} / 2\right.$; $\left.-l_{y} / 2<y<l_{y} / 2\right\}$. Let $\left(\boldsymbol{a}_{1}, \ldots, \boldsymbol{a}_{N}\right)$ denote a set of vortex locations in $R$, and $\left(m_{1}, \ldots, m_{n}\right)$ denote their respective orders which must all be positive. Let $M=\sum_{n=1}^{N} m_{n}$. Then, iff $l_{x} l_{y}>4 \pi M$, there exists a unique $f$ such that

(1) $f$ satisfies (1.7) in $R \backslash\left(\boldsymbol{a}_{1}, \ldots, \boldsymbol{a}_{N}\right)$.

(2) $f$ is periodic in $\mathbb{R}^{2}$, i.e., $f(x, y)=f\left(x+l_{x}, y\right)=f\left(x, y+l_{y}\right)$.

(3) $f \sim m_{n} \log \left|\boldsymbol{x}-\boldsymbol{a}_{n}\right|$ as $\left|\boldsymbol{x}-\boldsymbol{a}_{n}\right| \rightarrow 0 n=1, \ldots, N$. 
Proof To prove existence, we construct $f$ as the limit of the sequence of solutions of the following recursive set of boundary value problems:

$$
\begin{aligned}
& \nabla^{2} f_{0}-f_{0}=0 \quad \text { in } R \backslash\left(\boldsymbol{a}_{1}, \ldots, \boldsymbol{a}_{N}\right) \\
& \nabla^{2} f_{k}-f_{k}=\frac{1}{2}\left(e^{2 f_{k-1}}-2 f_{k-1}-1\right) \quad \text { in } R \backslash\left(\boldsymbol{a}_{1}, \ldots, \boldsymbol{a}_{N}\right) \forall k \geqslant 1 \\
& f_{k} \sim m_{n} \log \left|\boldsymbol{x}-\boldsymbol{a}_{n}\right| \quad \text { as }\left|\boldsymbol{x}-\boldsymbol{a}_{n}\right| \rightarrow 0 \quad \forall k, 1 \leqslant n \leqslant N
\end{aligned}
$$

$f_{k}$ is periodic $\forall k$.

It is easy to show that (2.1) is solvable for all $k$ : For $k=0$ the solution is given by

$$
f_{0}=-2 \pi \sum_{n=1}^{N} m_{n} G\left(\boldsymbol{x}, \boldsymbol{a}_{n}\right)
$$

in which $G$ denotes the Green's function for Helmholtz equation with periodic boundary conditions. Explicitly, $G$ is given by

$$
G(\boldsymbol{x}, \boldsymbol{y})=\frac{1}{2 \pi} \sum_{n_{1}=-\infty}^{\infty} \sum_{n_{2}=-\infty}^{\infty} K_{0}\left(\left|\boldsymbol{x}-\boldsymbol{y}-n_{1} l_{x} \boldsymbol{i}-n_{2} l_{y} \boldsymbol{j}\right|\right)>0,
$$

where $K_{0}$ denotes the $0^{\prime}$ th order modified Bessel function. Note that $G$ depends upon $l_{x}$ and $l_{y}$ and as $\min \left(l_{x}, l_{y}\right) \rightarrow \infty, G$ tends to $K_{0}$ pointwise. For $k \geqslant 1$ the solution is given by

$$
f_{k}=f_{0}-\frac{1}{2} \int_{R} G(\boldsymbol{x}, \boldsymbol{y})\left[e^{2 f_{k-1}}-2 f_{k-1}-1\right] .
$$

Since $e^{x}-x-1 \geqslant 0, f_{k}$ must be negative for all $k$. Furthermore, $\left\{f_{k}\right\}_{0}^{\infty}$ is monotonically decreasing for all $x \in R$, a fact which can be demonstrated by invoking inductive arguments. Let $\phi_{k}=f_{k+1}-f_{k}$. Clearly, $\phi_{k}$ satisfies

$$
\nabla^{2} \phi_{k}-\phi_{k}=\frac{1}{2}\left[\left(e^{2 f_{k}}-2 f_{k}\right)-\left(e^{2 f_{k-1}}-2 f_{k-1}\right)\right] .
$$

Thus, if we assume $f_{k}<f_{k-1}(<0)$, the right-hand-side of (2.5) must be positive. Hence, by the strong maximum principle we have $\phi_{k}<0$.

To prove convergence we need to show, yet, that $\left\{f_{k}\right\}_{k=0}^{\infty}$ is bounded from below. To this end we integrate $(2.1 b)$ over $R \backslash\left(\boldsymbol{a}_{1}, \ldots, \boldsymbol{a}_{N}\right)$ for $k=m+1$ to obtain

$$
\int_{R} \phi_{m}=\frac{1}{2}\left[l_{x} l_{y}-4 \pi M-\int_{R} e^{2 f_{m}}\right] .
$$

We focus attention first on the case $l_{x} l_{y}>4 \pi M$. Since $\phi_{m}<0$ we must have

$$
\int_{R} e^{2 f_{m}}>l_{x} l_{y}-4 \pi M \quad \forall m .
$$

Hence, the sequence $\left\{\int_{R} e^{2 f_{m}}\right\}_{0}^{\infty}$ must converge. The following conclusions immediately follow:

$$
\begin{aligned}
& \int_{R} \phi_{m} \underset{m \rightarrow \infty}{\longrightarrow} 0, \\
& \int_{R} e^{2 f_{m}} \underset{m \rightarrow \infty}{\longrightarrow} l_{x} l_{y}-4 \pi M .
\end{aligned}
$$


To prove, however, uniform convergence of $\left\{f_{k}\right\}_{0}^{\infty}$ it is necessary to utilize the Harnack inequality [12], together with (2.7). To show that Harnack inequality is indeed applicable in this case, we rewrite $(2.1 b)$ in the form

$$
\nabla^{2} f_{k}+h_{k} f_{k}=0
$$

where

$$
h_{k}=\frac{1}{2} \frac{1-e^{2 f_{k}}+2\left(f_{k-1}-f_{k}\right)}{f_{k}} ;
$$

since $f_{k-1}<f_{k}<0$, we have $-2<h_{k}<0 \forall k$. By (2.7),

$$
\sup _{x \in R} f_{k} \geqslant C>-\infty \quad \forall k,
$$

where $C$ is independent of $k$. Hence, from Harnack inequality it follows that

$$
\inf _{\boldsymbol{x} \in R_{\epsilon}} f_{k} \geqslant A_{\epsilon} \sup _{\boldsymbol{x} \in R_{\epsilon}} f_{k}>A_{\epsilon} C>-\infty,
$$

in which

$$
\begin{aligned}
& R_{\epsilon}=R \backslash \bigcup_{n=1}^{N} B\left(\boldsymbol{a}_{n}, \epsilon\right), \\
& B\left(\boldsymbol{a}_{n}, \epsilon\right)=\left\{\boldsymbol{x}|| \boldsymbol{x}-\boldsymbol{a}_{n} \mid \leqslant \epsilon\right\},
\end{aligned}
$$

and $A_{\epsilon}$ is independent of $k$. Consequently, $\left\{\inf _{x \in R_{\epsilon}} f_{k}\right\}_{k=0}^{\infty}$ is bounded from below for all positive $\epsilon$. Thus, $\left\{f_{k}\right\}_{k=0}^{\infty}$ must converge, or, $f_{k} \underset{k \rightarrow \infty}{\longrightarrow} f$ uniformly in $R_{\epsilon}$ and pointwise in $R \backslash\left(\boldsymbol{a}_{1}, \ldots, \boldsymbol{a}_{N}\right)$.

To show that $f$ is indeed the desired periodic solution of (1.7) it is necessary to demonstrate that it has the required logarithmic behaviour near the vortices, i.e.

$$
|f| \leqslant b+\left|m_{n} \log \right| \boldsymbol{x}-\boldsymbol{a}_{n}|| \quad \forall \boldsymbol{x} \in \bigcup_{n=1}^{N} B\left(\boldsymbol{a}_{n}, \epsilon\right) \quad 1 \leqslant n \leqslant N,
$$

which can be written in the alternative form $\left|f-f_{0}\right|<\tilde{b}$. We prove (2.8) invoking inductive arguments. We assume by induction that $\left|f_{k-1}-f_{0}\right| \leqslant b_{k-1} \mid$ (an assumption which is clearly valid for $k=1$ ). Then, utilizing (2.4), we obtain

$$
\left|f_{k}-f_{0}\right| \leqslant b_{k}=\chi_{1}(\epsilon)+\chi_{2}(\epsilon)+\eta(\epsilon) b_{k-1},
$$

where

$$
\begin{gathered}
\chi_{1}(\epsilon)=\frac{1}{2} \sup _{\boldsymbol{x} \in R \backslash R_{\epsilon}} \int_{R_{\epsilon}} G(\boldsymbol{x}, \boldsymbol{y})\left(e^{2 f}-2 f-1\right) d y, \\
\chi_{2}(\epsilon)=-\sup _{\boldsymbol{x} \in R \backslash R_{e}} \sum_{n=1}^{N} \int_{B\left(\boldsymbol{a}_{n}, \epsilon\right)} G(\boldsymbol{x}, \boldsymbol{y}) m_{n} \log \left|\boldsymbol{y}-\boldsymbol{a}_{n}\right| d y,
\end{gathered}
$$

and

$$
\eta(\epsilon)=\sup _{\boldsymbol{x} \in R \backslash R_{\epsilon}} \sum_{n=1}^{N} \int_{B\left(\boldsymbol{a}_{n}, \epsilon\right)} G(\boldsymbol{x}, \boldsymbol{y}) d y .
$$


For sufficiently small $\epsilon$ we have $\eta<1$. Hence, we can take the limit $k \rightarrow \infty$ in $(2.9 a)$ to obtain

$$
\left|f-f_{0}\right| \leqslant b=\frac{\chi_{1}(\epsilon)+\chi_{2}(\epsilon)}{1-\eta(\epsilon)},
$$

which completes the proof of (2.8).

Taking the limit $k \rightarrow \infty$ in (2.4) yields, in view of (2.8),

$$
f=f_{0}-\frac{1}{2} \int_{R} G(\boldsymbol{x}, \boldsymbol{y})\left[e^{2 f}-2 f-1\right],
$$

from which it can be readily verified that $f$ is indeed a periodic solution of (1.7).

To prove uniqueness, we suppose that two periodic solutions of (1.7), $f$ and $\tilde{f}$, coexist in $R$ for the same set of vortex locations and orders. Then, $\phi=f-\tilde{f}$ is periodic as well, regular in $R$, and satisfies

$$
\nabla^{2} \phi+\left[\frac{1}{2} e^{2 f} \frac{1-e^{2 \phi}}{\phi}\right] \phi=0 .
$$

By the strong maximum $\phi$ can have neither a positive maximum nor a negative minimum. Hence, since $\phi$ is periodic it must vanish identically.

Finally, we consider the case $l_{x} l_{y} \leqslant 4 \pi M$. Integration of (1.7) over $R$ yields

$$
\int_{R} e^{2 f}=l_{x} l_{y}-4 \pi M
$$

Hence, if $l_{x} l_{y} \leqslant 4 \pi M$ no periodic solution for (1.7) can exist.

It should be mentioned that the same method can be used to prove the existence of Taubes' [8] arbitrary $N$-vortex solution in $\mathbb{R}^{2}$. If instead of prescribing (2.1d) we require $\lim _{|x| \rightarrow \infty} f_{k}=0$, the sequence $\left\{f_{k}\right\}_{0}^{\infty}$ is once again both negative as well as monotonically decreasing. Then, integrating (2.1b) over $\mathbb{R}^{2}$, we obtain, since $f_{k}<f_{k-1}$, the inequality

$$
\int_{\mathbb{R}^{2}}\left(1-e^{2 f_{k-1}}\right) \leqslant 4 \pi M
$$

from which, together with Harnack inequality, follows the existence of Taubes' solution.

\section{The large cell limit}

In the following we investigate the behaviour of the solution in the limit when both $l_{x}$ and $l_{y}$ tend to infinity. Intuitively, we expect that the limit would be identical with Taubes' [8] arbitrary $\mathrm{N}$-vortex solution, which is exactly what the following theorem states.

Theorem 2 The limit function $g=\lim _{l_{x}, l_{y} \rightarrow \infty} f$ exists and satisfies

$$
\begin{aligned}
& \nabla^{2} g-\frac{1}{2}\left(e^{2 g}-1\right)=0 \quad \text { in } \mathbb{R}^{2} \backslash\left(\boldsymbol{a}_{1}, \ldots, \boldsymbol{a}_{N}\right) \\
& g \sim m_{n} \log \left|\boldsymbol{x}-\boldsymbol{a}_{n}\right| \quad \text { as }\left|\boldsymbol{x}-\boldsymbol{a}_{n}\right| \rightarrow 0 \quad \forall 1 \leqslant n \leqslant N \\
& g \rightarrow 0 \quad \text { as }|\boldsymbol{x}| \rightarrow \infty
\end{aligned}
$$


Proof We first demonstrate that $f^{l} \stackrel{\text { def }}{=} f\left(\boldsymbol{x}, l_{x}, l_{y}, \boldsymbol{a}_{1}, \ldots, \boldsymbol{a}_{N}\right)$ possesses a certain monotonicity property. Consider, then, the function $f^{3 l} \stackrel{\text { def }}{=} f\left(\boldsymbol{x}, 3 l_{x}, 3 l_{y}, \boldsymbol{a}_{1}, \ldots, \boldsymbol{a}_{N}\right)$. Both $f^{l}$ and $f^{3 l}$ are periodic on the boundaries of the rectangle

$$
R^{3 l}=\left\{(x, y) \mid-\frac{3}{2} l_{x} \leqslant x \leqslant \frac{3}{2} l_{x} ; \quad-\frac{3}{2} l_{y} \leqslant y \leqslant \frac{3}{2} l_{y}\right\} .
$$

Yet $f^{3 l}$ is singular only at $\boldsymbol{a}_{1}, \ldots \boldsymbol{a}_{N}$, whereas $f^{l}$ is singular at $\boldsymbol{a}_{i} \pm \alpha l_{x} \boldsymbol{i} \pm \beta l_{y} \boldsymbol{j}$, where $1 \leqslant i \leqslant N$ and $\alpha$ and $\beta$ may take the values 0 and 1 . Thus, by comparison theorems we have $f^{3 l}>f^{l}$. Therefore, $\left\{f^{3^{k} l}\right\}_{n=0}^{\infty}$ is a monotonically increasing sequence of negative functions, and hence, must converge pointwise. Furthermore,

$$
f^{3^{n} l} \leqslant f_{0}^{3^{n} l} \Rightarrow g \leqslant-\sum_{m=1}^{N} \frac{1}{2 \pi} K_{0}\left(\left|\boldsymbol{x}-\boldsymbol{a}_{m}\right|,\right.
$$

where $f_{k}^{3^{n} l}$ denotes the solution of $(2.1 b)$ in $R^{3^{n} l}$. Therefore, $g$ has the the same asymptotic behaviour as $f$ near the vortices, and hence, $\left|f^{3^{n} l}-g\right| \rightarrow 0$ uniformly on any compact set in $\mathbb{R}^{2}$.

To prove that the limit indeed satisfies (3.1), it is first necessary to show that $\left|f^{3^{n} l}\right|$ is uniformly bounded in $R^{3^{n} l}$ (except near the vortices) as $n \rightarrow \infty$. It is clear that $\left|f^{3^{n} l}\right|<\left|f^{l}\right|$ everywhere in $R^{3^{n} l}$. However, while $f^{3^{n} l}$ is singular there at $N$ different points, $f^{l}$ possesses $3^{n} N$ singularities. It is, therefore, necessary to show that $\left|f^{3^{n} l}\right|$ is bounded in $R^{3^{n} l} / R^{l}$ where $f^{3^{n} l}$ is regular but $f^{l}$ has singularities.

We prove that boundedness property by induction. Clearly, by (2.2) and (2.3),

$$
\left|f_{0}^{3^{n} l}\right| \leqslant C_{0} \quad \text { in } R^{3^{n} l} / R^{l}
$$

where $C_{0}$ is independent of $n$. Assume now that $\sup _{\boldsymbol{x} \in R^{3^{n} l}}\left|f_{k}^{3^{n} l}-f_{0}^{3^{n} l}\right| \leqslant C_{k}^{n}$, then by (2.4) $\sup _{x \in R^{3^{n} l}}\left|f_{k+1}^{3^{n} l}-f_{0}^{3^{n} l}\right| \leqslant C_{k+1}^{n}$ and

$$
C_{k+1}^{n}=\sup _{x \in R_{\epsilon}^{l}}\left|f^{l}\right|+\alpha_{n}(\epsilon)\left(C_{k}^{n}+C_{0}\right) \beta(\epsilon)
$$

in which $\epsilon>0$,

$$
\begin{gathered}
R_{\epsilon}^{l}=R^{l} \backslash \bigcup_{m=1}^{N} B\left(\boldsymbol{a}_{m}, \epsilon\right), \\
\alpha_{n}(\epsilon)=\int_{\bigcup_{m} B\left(\boldsymbol{a}_{m}, \epsilon\right)} G^{n}(\boldsymbol{x}, \boldsymbol{y}) d y,
\end{gathered}
$$

and

$$
\beta(\epsilon)=\sum_{m=1}^{N} \int_{B\left(\boldsymbol{a}_{m}, \epsilon\right)} G^{0}(\boldsymbol{x}, \boldsymbol{y})\left|f_{0}^{l}-C_{0}\right| d y,
$$

where the union in $(3.2 c)$ is over all the vortices of $f^{l}$ in $R^{3^{n} l}$ and $G^{n}$ denotes the Helmholtz equation Green's function (2.3) in $R^{3^{n} l}$.

For sufficiently small $\epsilon \leqslant \epsilon_{0}$ we have $\alpha_{n}(\epsilon)<\alpha<1$ for all $n$ (note that the integral of $G$ over $R^{3^{n} l}$ is unity). Hence, by $(3.2 a) C_{k}^{n}<\bar{C} /(1-\alpha)$ for all $k$ and $n$, from which uniform boundedness of $f^{3^{n} l}$ in $R^{3^{n} l} / R^{l}$ immediately follows. 
Denote the limit, which must be bounded in $\mathbb{R}^{2} \backslash R^{l}$, by $g$. Then,

$$
\begin{gathered}
\left|\int_{R^{3^{n l}}} G(\boldsymbol{x}, \boldsymbol{y})\left(\exp \left\{2 f^{3^{n} l}\right\}-2 f^{3^{n l}}\right) d y-\frac{1}{2 \pi} \int_{\mathbb{R}^{2}} K_{0}(|\boldsymbol{x}-\boldsymbol{y}|)(\exp \{2 g\}-2 g) d y\right| \\
\leqslant \int_{\mathbb{R}^{2} \backslash R^{3^{n l}}} \frac{1}{2 \pi} K_{0}(|\boldsymbol{x}-\boldsymbol{y}|)(\exp \{2 g\}-2 g) d y+\int_{R^{3^{n l}}}\left(G-\frac{1}{2 \pi} K_{0}\right)\left(\exp \left\{2 f^{3^{n} l}\right\}-2 f^{3^{n l}}\right) d y \\
\quad+\int_{R^{3^{n l}}} \frac{1}{2 \pi} K_{0}(|\boldsymbol{x}-\boldsymbol{y}|)\left|\left(\exp \left\{2 f^{3^{n} l}\right\}-2 f^{3^{n} l}\right)-(\exp \{2 g\}-2 g)\right| d y .
\end{gathered}
$$

From the boundedness of $f^{3^{n} l}$ and $g$ it follows that the first two terms on the right-hand side of (3) converge pointwise to zero as $n \rightarrow \infty$. For the third term we have

$$
\begin{gathered}
\int_{R^{3^{n l}}} \frac{1}{2 \pi} K_{0}(|\boldsymbol{x}-\boldsymbol{y}|)\left|\left(\exp \left\{2 f^{3^{n} l}\right\}-2 f^{3^{n l} l}\right)-(\exp \{2 g\}-2 g)\right| d y \\
\leqslant \sup _{|\boldsymbol{x}| \leqslant r}\left|\left(\exp \left\{2 f^{3^{n l} l}\right\}-2 f^{3^{n l} l}\right)-(\exp \{2 g\}-2 g)\right| \\
+\sup _{|\boldsymbol{x}| \in R^{3^{n l}}}\left|\left(\exp \left\{2 f^{3^{n} l}\right\}-2 f^{3^{n} l}\right)-(\exp \{2 g\}-2 g)\right| \int_{|\boldsymbol{y}| \geqslant r} \frac{1}{2 \pi} K_{0}(|\boldsymbol{x}-\boldsymbol{y}|) d y .
\end{gathered}
$$

The first term on the right-hand side of the above inequality vanishes, as $n \rightarrow \infty$, in view of the uniform convergence of $\left|f^{3^{n} l}-g\right|$ to 0 in $B(0, r)$. The second term on the right-hand side of (3) can be set arbitrarily small for sufficiently large $r$ in view of the boundedness of $f^{3^{n} l}$ and $g$.

Taking the limit $n \rightarrow \infty$ in $(2.10)\left(f=f^{3^{n} l}\right)$, we obtain

$$
g=-\frac{1}{2 \pi} \sum_{n=1}^{N} K_{0}\left(\left|\boldsymbol{x}-\boldsymbol{a}_{n}\right|\right)-\frac{1}{4 \pi} \int_{\mathbb{R}^{2}} K_{0}(|\boldsymbol{x}-\boldsymbol{y}|)\left(e^{2 g}-2 g-1\right) d y,
$$

from which it follows immediately that $g$ must satisfy both (3.1a) and (3.1b). Yet it is still necessary to show that $g$ must decay as $|\boldsymbol{x}| \rightarrow \infty$. We notice that for sufficiently large $n$

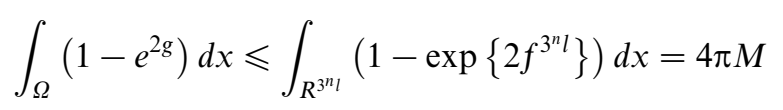

for any $\Omega \subset \mathbb{R}^{2}$. Hence, the integral of $1-\exp \{2 g\}$ over $\mathbb{R}^{2}$ must exist. In fact, in view of (3.5),

$$
\int_{\mathbb{R}^{2}}\left(1-e^{2 g}\right) d x=4 \pi M
$$

Consequently, $g$ must satisfy (3.1c) as well, and is, therefore, identical with Taubes' [8] solution (note that the foregoing discussion may serve as another existence proof for Taubes' solution).

It is still necessary to show that

$$
\lim _{l_{x}, l_{y} \rightarrow \infty} f=g .
$$


Let $l_{x}^{k}, l_{y}^{k} \uparrow \infty$ as $k \rightarrow \infty$, and $R_{k}=R\left(l_{x}^{k}, l_{y}^{k}\right)$. Clearly, $f\left(l_{x}^{k}, l_{y}^{k}\right) \leqslant g$ everywhere in $R_{k}$ since $f\left(3^{n} l_{x}^{k}, 3^{n} l_{y}^{k}\right) \uparrow g$ as $n \rightarrow \infty$. Hence,

$$
\begin{aligned}
\lim _{k \rightarrow \infty} \int_{R_{k}} \mid \exp \left\{2 f\left(l_{x}^{k}, l_{y}^{k}\right)\right\} & -\exp \{2 g\} \mid d x \\
& =\lim _{k \rightarrow \infty} \int_{R_{k}}\left(1-\exp \left\{2 f\left(l_{x}^{k}, l_{y}^{k}\right)\right\}\right)-(1-\exp \{2 g\}) d x=0 .
\end{aligned}
$$

The above $L_{1}^{\text {loc }}$ convergence of $e^{2 f}$ to $e^{2 g}$ implies $L_{1}^{\text {loc }}$ convergence of $f$ to $g$ in view of the uniform boundedness of $f$ with respect to $l_{x}$ and $l_{y}$ in every sub-domain of $R$ from which some neighbourhood of the vortices is excluded.

It is, therefore, clear that $f$ converges pointwise to $g$ almost everywhere. To show that the convergence is uniform on every compact set from which some neighbourhood of the vortices is excluded, we demonstrate that $|\nabla f|$ is uniformly bounded with respect to $l_{x}$ and $l_{y}$ on such a set. To this end we take the gradient of (2.10) to obtain

$$
|\nabla f| \leqslant\left|\nabla f_{0}\right|+\int_{R}\left|\nabla_{x} G(\boldsymbol{x}, \boldsymbol{y})\right||f| d y,
$$

and since

$$
|f| \leqslant C-\sum_{n=1}^{N} m_{n} \log \left|\boldsymbol{x}-\boldsymbol{a}_{n}\right|,
$$

the necessary boundedness property is easily derived.

\section{Conclusion}

We have demonstrated, in $\S 2$, the existence of a unique periodic solution to the first order Ginzburg-Landau equations with periods $l_{x}$ and $l_{y}$ in the respective $x$ and $y$ directions, for any given set of vortex locations and orders within the fundamental cell and whenever $l_{x} l_{y}>4 \pi M, M$ being the topological invariant. When $l_{x} l_{y} \leqslant 4 \pi M$ no such solution exists. These results have previously been proved by Noguchi [10] by applying direct methods of the calculus of variations. Our method of proof is based on an iterative scheme which proves to be very useful in $\S 3$. Linear bifurcation analysis $[3,1,2]$ finds the periodic superconducting solution which bifurcates from the normal state $(\psi=0)$, shows that the area of the unit cell at the bifurcation is given by $l_{x} l_{y}=2 \pi M / \kappa^{2}$. Odeh [5] proved, in addition, that periodic weak solutions exist whenever $l_{x} l_{y} \geqslant 2 \pi M / \kappa^{2}$. Both results are in accordance, for $\kappa=1 / \sqrt{2}$ with ours. The results are, however, stronger in the critical case, since they provide information on the manner according to which vortices are arranged within the fundamental cell in the highly non-linear regime. In addition a non-existence result is derived as well.

Besides proving existence of periodic solutions we have also investigated, in $\S 3$, their behaviour in the large cell limit, when both $l_{x}$ and $l_{y}$ tend to infinity. We proved, using estimates derived from the iterative scheme in $\$ 2$, that in that limit the periodic solutions tend, for any set of vortices, to Taubes' arbitrary $N$-vortex solution in $\mathbb{R}^{2}$ corresponding to the same set of vortices [8]. This result can serve as an alternative proof for the existence of Taubes' solution. Likewise, the method in $\$ 2$ can be utilized with slight modification 
in order to prove the existence of Taubes' solution as well. It is conjectured in Chapman et al. [6] that, as the size of the fundamental cell increases, $|\psi| \rightarrow 1$ except in the close neighbourhood of the vortices. Our results demonstrate the validity of this conjecture for the critical case.

The periodic solutions for the critical case are quite different from those obtained for the more general case when $\kappa>1 / \sqrt{2}$. For the latter case, linear bifurcation analysis indicates that a countable number of possible vortex arrangements inside the fundamental cell can exist, whereas for the critical case the vortices can be arbitrarily arranged. A similar situation exist while seeking an arbitrary $N$-vortex solution in $\mathbb{R}^{2}$. Such a solution exists for the critical case, whereas for $\kappa>1 / \sqrt{2}$ it is conjectured in [6] that the only steady vortex solution to the Ginzburg-Landau equations is that of an isolated vortex.

Another peculiarity of the periodic solutions in the critical case is that their energy depends neither on the size of the cell, nor on its shape. The energy functional may be written in the present scaling, for $\kappa=1 / \sqrt{2}$, in the form [8]

$$
J=\int_{R}\left(\frac{1}{2}-|\psi|^{2}+\frac{|\psi|^{4}}{2}+|\boldsymbol{H}|^{2}+|\sqrt{2} \nabla \psi-i \boldsymbol{A} \psi|^{2}\right) d x d y .
$$

Then, rearranging the integrand yields

$$
J=\int\left[J_{1}^{2}+J_{2}^{2}+J_{3}^{2}+\nabla \times\left(\sqrt{2}|\psi|^{2} \boldsymbol{A}+i\left(\psi^{*} \nabla \psi-\psi \nabla \psi^{*}\right)\right) \cdot \hat{\boldsymbol{k}}+\sqrt{2} H\right] d x d y,
$$

where $J_{1}, J_{2}$, and $J_{3}$ are the respective right hand sides of $(1.2 a),(1.2 b)$ and (1.2c). Utilizing $(1.1 b)$, we may simplify the integrand even further, for any solution of (1.2) to obtain

$$
J=\int_{R}[\nabla \times \nabla \times \boldsymbol{H}+\sqrt{2} H] d x d y .
$$

Periodicity of $\boldsymbol{H}$ together with (1.6) then yields $J=4 \pi M$. The latter result is independent of the fundamental cell size and shape as well as of the vortices arrangement within the cell (cf. also Jacobs et al. [13]).

Finally, it seems worth mentioning in that context that no self-duality solution can exist in cases where both negative and positive vortex orders are present. Smoothness of $\boldsymbol{A}$ at the vortices requires that the magnetic field at a negative vortex would be opposite directed with respect to its orientation near a positive one. Hence, (1.2) cannot be satisfied near a negative vortex, and a different set of equations should be satisfied there [8], namely

$$
\begin{gathered}
\sqrt{2} \frac{\partial \psi_{1}}{\partial x}+A_{x} \psi_{2}+\sqrt{2} \frac{\partial \psi_{2}}{\partial y}-A_{y} \psi_{1}=0 \\
\sqrt{2} \frac{\partial \psi_{1}}{\partial y}+A_{y} \psi_{2}-\sqrt{2} \frac{\partial \psi_{2}}{\partial x}+A_{x} \psi_{1}=0 \\
H-\frac{1}{\sqrt{2}}\left(\psi_{1}^{2}+\psi_{2}^{2}-1\right)=0 .
\end{gathered}
$$

Applying the same procedure following (1.2), equation (1.7) is obtained once again. Hence, (1.7) must be satsified everywhere.

Suppose now that a self-duality solution in the presence of both positive and negative vortices can exist. In view of $(1.2 c)$ and (4.1c), a curve in $\mathbb{R}^{2}$ along which $H=0$ must exist 
as well. Denote this curve by $\Gamma$. Along $\Gamma$ we have by $(1.2 c) f=0$. Furthermore, since $f$ is negative near $\Gamma, \partial f / \partial n$ must vanish on it as well. This is impossible, however, since any solution of (1.7) which is negative near $\Gamma$ and vanishes on it, must have a non-vanishing normal derivative on each point of $\Gamma[12]$.

\section{References}

[1] Abrikosov, A. A. (1957) On the magnetic properties of superconductors of the second group. Soviet Phys. JETP 5, 1175-1204.

[2] Kleiner, W. W., Roth, L. M. \& Autler, S. W. (1964) Bulk solution of Ginzburg-Landau equations for type II superconductors. Phys. Rev. 133, 1226-1227.

[3] Chapman, S. J. (1994) Nucleation of superconductivity in decreasing fields I. Euro J. Appl. Math. 5, 449-468.

[4] Essmann, U. \& Träuble, H. (1967) The direct observation of individual flux lines in Type II superconductors. Phys. Lett. A24, 526-527.

[5] OdeH, F. (1967) Existence and bifurcation theorems for the Ginzburg-Landau equations. J. Math. Phys. 8, 2351-2356.

[6] Chapman, S. J., Howison, S. D. \& Ockendon, J. R. (1992) Macroscopic models for superconductivity. SIAM Rev. 34, 529-560.

[7] Bogomolnyi, E. (1976) The stability of classical solutions. Sov. J. Nucl. Phys. 24, 449-454.

[8] Taubes, C. H. (1980) Arbitrary $N$-vortex solutions to the first order Ginzburg-Landau equations. Commn. Math. Phys. 72, 277-292.

[9] ALmog, Y (1998) Arbitrary $N$-vortex self-duality solutions to the Ginzburg-Landau equations satisfying normal state conditions at infinity. Asymptotic Analysis 17, 267-278.

[10] NoGUCHI, M. (1987) Young-Mills-Higgs theory on a compact Riemann surface. J. Math. Phys. 28, 2343-2346.

[11] Yang, Y. (1991) Vortices on asymptotically Euclidean Riemann surfaces. Nonlinear Anal. 15, $577-596$.

[12] Protter, M. H. \& Weinberger, H. F. (1967) Maximum Principles in Differential Equations. Prentice-Hall.

[13] Jacobs, A. E., Mukamel, D. \& Walker, M. B. (1981) Critical points of infinite order in charge-wave-density and in superconductors. Phys. Rev. B. 24, 5195-5203. 